\title{
Blood count parameters in the course of multiple sclerosis
}

\author{
Weronika Kasprzycka1 ${ }^{\oplus}$, Magdalena Nieśpiałowska ${ }^{2} \oplus$, Beata Jakubowska-Solarska ${ }^{2} \oplus$ \\ ${ }^{1}$ Chair and Department of Human Physiology, I Faculty of Medicine with Dentistry Division, \\ Medical University of Lublin \\ ${ }^{2}$ Department of Hematological Diagnostics, Faculty of Pharmacy with Medical Analytics Division, \\ Medical University of Lublin
}

\begin{abstract}
Summary
Background. Multiple sclerosis (MS) is the most common demyelinating disease of the central nervous system (CNS). It mostly affects young people. Pathological lesions cause destruction of myelin sheath around axons and impede transmission of nerve impulses in CNS. The diagnosis of MS is based on clinical evaluation, biochemical blood tests and cerebrospinal fluid tests as well as on imaging. The study aim was assessment of blood counts of MS patients.
\end{abstract}

Materials and methods. The study group comprised 189 people (77 healthy) and $112 \mathrm{MS}$ patients treated at the Department of Neurology of the Medical University of Lublin. Parameters of EDTA anticoagulated whole blood were determined on the Advia 2012i analyzer. Statistica 12.5 program was used for statistical analysis.

Results. Patients evaluated with regard to clinical condition and disease progression demonstrate differences in RBC count, hemoglobin, hematocrit and mean corpuscular volume (MCV). RBC count of patients with relapsing-remitting MS (RRMS) was lower (Me $=4.73$ million/ $\mu l)$ than for patients with secondary progressive MS (SPMS) $(M e=5.03$ million $/ \mu l)$. Additionally, differences in hemoglobin level were observed between RRMS patients (Me = $13.9 \mathrm{~g} / \mathrm{dl})$ and $S P M S$ patients $(M e=14.7 \mathrm{~g} / \mathrm{dl})$. Significant differences were also observed in hematocrit; (Me $=40.5 \%)$ for RRMS and (Me = 44\%) for SPMS patients. Differences in $M C V$ between the examined groups of MS patients and the control group were not statistically

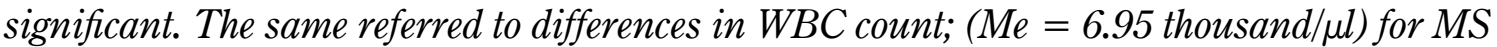
patients and (Me $=6.59$ thousand/ $\mu$ l) for control group as well as platelet count; (Me $=237.5$

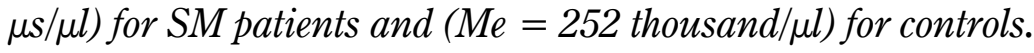

Conclusion. Analysis of blood parameters reveals significant differences between MS patients and controls as well as differences between RRMS and SPMS patients with regard to red blood cells. An in-depth analysis also in terms of disease duration and stage of clinical advancement may be a valuable source of information on the overall condition of MS patients.

Key words: multiple sclerosis, blood count, RBC, WBC, PLT, hemoglobin

J. Transf. Med. 2019; 12: 117-123

Correspondence address: Weronika Kasprzycka, Chair and Department of Human Physiology, I Faculty of Medicine with Dentistry Division, Medical University of Lublin, 20-080 Lublin, e-mail: weronikakasprzycka2@gmail.com 


\section{Introduction}

Multiple sclerosis is an example of a neurodegenerative disease leading to progressive damage of neurons and patient's disability. The incidence rate of MS is growing and in Poland the number of MS cases is estimated at more than 40 thousand with 2000 new cases recognized each year. The main age group affected are people between 20 and 40 years of age [1]. MS diagnosed before the age of 16 is generally defined as pediatric-onset MS while MS recognized after the age of 50 is defined as late, adult-onset MS. The latter is associated with worse prognosis, rapid progress and more severe symptoms. Women are diagnosed with MS twice as often as men, which is related to higher estrogen levels [2, 3]. It is estimated that MS can shorten the life expectancy by 6-7 years on average. Death may occur as result of neurological complications and immobilization [4-6]. The MS incidence is also correlated with latitude; the further the distance from the Equator, the higher the MS risk. This may be explained by reduced exposure to sunlight and reduced vitamin D synthesis, which acts as immunomodulating agent. It has also been reported that MS is more common in people with light skin pigmentation $[7,8]$.

Multiple sclerosis is a chronic disease that affects the central nervous system (CNS). It presents with demyelination lesions in the brain and spinal cord, by inflammation processes, degradation of axonal myelin sheaths and destruction of glial sheaths [9]. As a result, the electrical impulses from the brain do not flow smoothly to the target nerve which is the underlying cause of MS. Clinical symptoms of the disease vary depending on the demyelination site. The most common include: muscle weakness, numbness, tingling, stiffness, vision problems, dizziness, impaired balance and coordination $[10,11]$. At a later stage of the disease the patient often experiences spasticity and muscle spasms. Lesions within the spinal cord may lead to bladder and bowel disorders $[12,13]$. Other possible paroxysmal symptoms are: Lhermitte's sign (upon movement of the neck a person may experience an electric shock-like sensation) and Uhthoff's syndrome (temporary blurred vision after physical activity, fever, or hot bath). A typical course of MS is marked by relapses (worsening of neurological symptoms or occurrence of new 24 -hour episodes) followed by remissions (the intervals between relapses when symptoms improve or disappear). The average number of relapses is specific for each patient. Patients may be grouped into four major categories based on the course of the disease: Relapsing-Remitting (RRMS), Secondary Progressive (SPMS), Primary Progressive
(PPMS) and Progressive Relapsing (PRMS) [14-16]. During the course of the disease inflammatory infiltrates are observed in histopathological imaging as sign of the defensive reaction of the organism. MS is therefore said to be an autoimmune inflammatory neurological disease [17].

\section{General aim}

The diagnostic procedure of MS is difficult due to multifactorial etiology of the disease, multitude of pathological processes that occur and variety of clinical types of the disease. The final diagnosis is often delayed. The recognition of MS is based on: evaluation of the patient's clinical status, imaging, biochemical blood tests and cerebrospinal fluid (CSF) analysis [18-20]. Up to date no specific and sensitive marker for early diagnosis of MS has been found. It is therefore essential to conduct research in various medical disciplines to be able to successfully treat patients with MS. Assessment of patients' blood parameters in the course of the disease gives the chance to gain more information on the disease as well as shows the effect of MS on the patient's organism [21]. The latest publications [22-24] point to the fact that simple indicators such as: neutrophil-to-lymphocyte ratio (NLR),monocyte-to-lymphocyte ratio (MLR) and platelet-to-lymphocyte ratio (PLR) may be promising markers in assessment of inflammatory response or immunization. The aim of this study was to perform a comparative evaluation of blood parameters of patients diagnosed with MS and in healthy volunteers, as well as to demonstrate that NLR, MLR and PLR may have a significant role to play in the process of MS diagnosis.

\section{Materials and methods}

The study comprised a group of 189 people; 77 were healthy volunteers (30 males and 47 females) aged 19-86 years and 112 were MS patients aged 18-86 years (average 42 years) of which: 62 were females (55.35\%) and 50 were males $(44,65 \%)$. The patients were treated in the Department of Neurology of the $4^{\text {th }}$ Clinical Hospital in Lublin in the period 2012 and 2016. Various types of MS were represented and the average duration of the disease was 4 years.

The largest group $(67-59.82 \%)$ were patients diagnosed with Relapsing-Remitting MS, then a group diagnosed with Primary Progressive MS $(21-18.75 \%)$. Seven $(7-6.25 \%)$ patients were diagnosed with Secondary Progressive MS. Clinically Isolated Syndrome (CIS) was diagnosed in 17 patients (15.18\%) Figure 1. Level of disability was measured by the Expanded Disability Status 


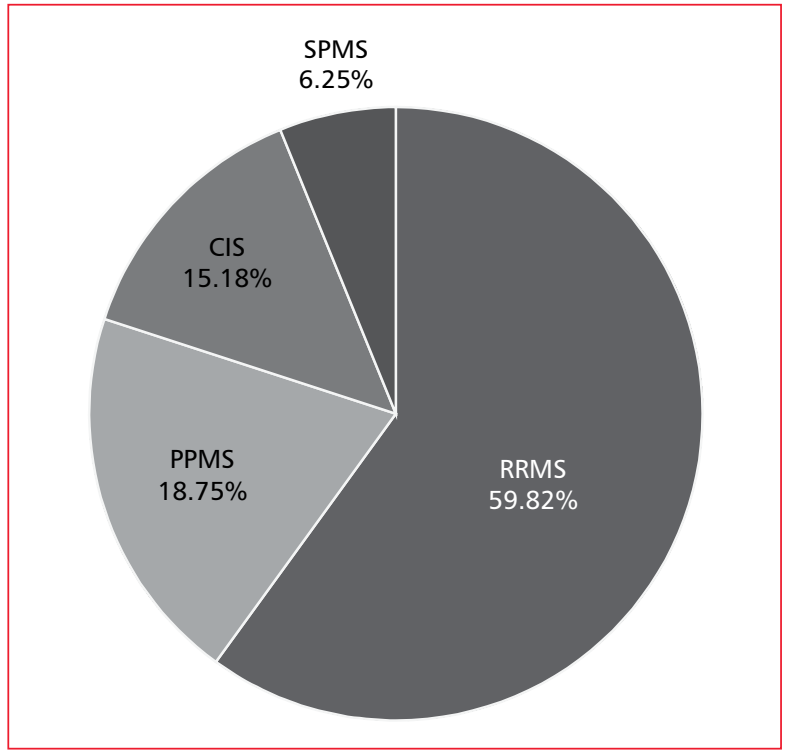

Figure 1. Percentage of MS patients with various types of the disease

Scale (EDSS). Mildly disabled patients (1-3.5 EDSS) were $68.7 \%$ of all patients. $21.4 \%$ presented moderate disability (4-5 EDSS). The remaining (9.9\%) were assigned an EDSS score of 6 to 9.

Research material in both groups (healthy volunteers and MS patients) the was EDTA anticoagulated whole blood collected from fasting patients according to standard procedures. Blood count parameters were analyzed by Advia $2120 \mathrm{i}$ analyser.The patients' medical histories provided information on: type of MS, EDSS score and duration of disease. STATISTICA 12.5 software was used for statistical analysis of data.

\section{Results}

The first stage of analysis included descriptive statistics of quantitative and qualitative data. Data distribution analysis was then performed with Shapiro-Wilk test. The results were out of the normal distribution $(\mathrm{p}<0.05)$ which affected the selection of tests for the further stage of the statistical analysis. Comparisons between the study and control groups were performed using the Mann-Whitney U test. Comparisons of blood parameters between groups of patients with different types of MS and different degrees of disability were made using the Kruskal-Wallis test. The next stage of analysis was to perform calculations of NLR, MLR and PLR which were then compared with results for the control group.

Table 1 presents the average blood parameters for the study group and control group obtained using the Spearman's Rank Correlation test.

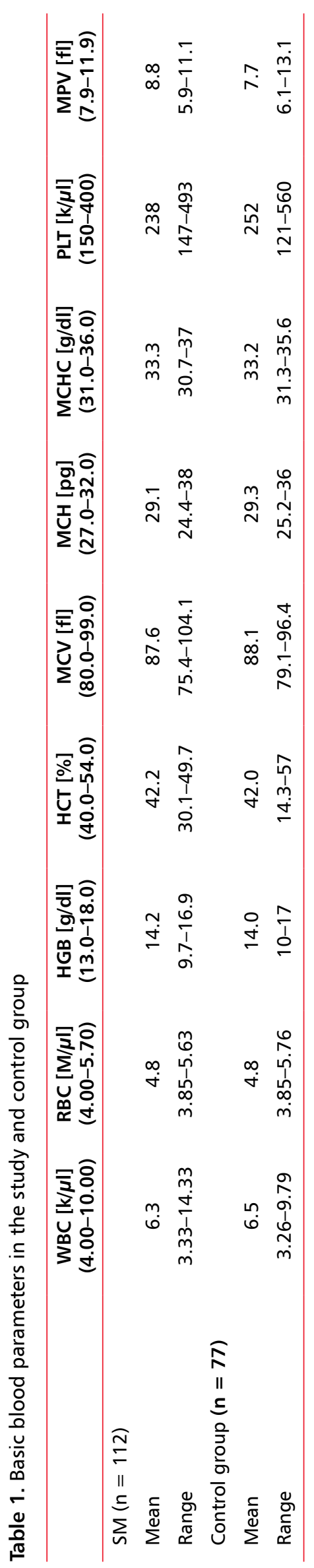


The MPV value was significantly higher in the study group as compared to the control group $(\mathrm{p}=0.001)$. Results are shown in Figure 2.

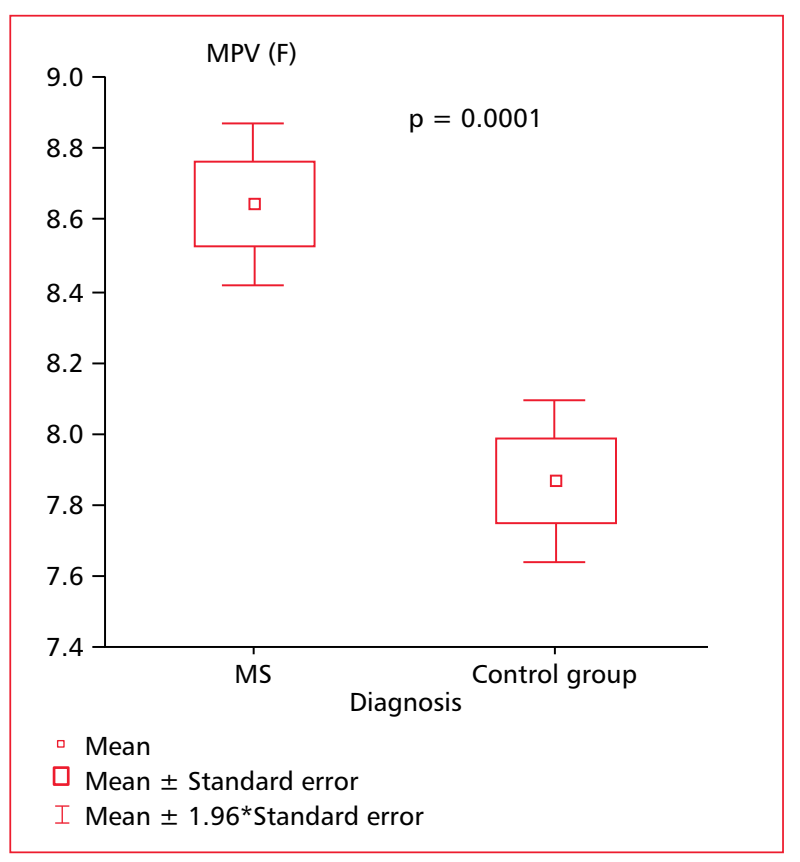

Figure 2. MPV level in serum of MS patients and in the control group. Mann-Whitney $U$ test
The analysis of basic blood parameters in groups of patients with different types of MS was performed with Kruskall-Wallis test (Table 2). Significant differences in $\mathrm{WBC}$ and $\mathrm{RBC}$ values were found between groups of patients suffering from various types of MS. The lowest WBC value was recorded for patients with Relapse-Remitting MS $(5.91 \mathrm{k} / \mu \mathrm{l})$, while the highest in patients with Primary Progressive MS $(7.10 \mathrm{k} / \mu \mathrm{l})$. The lowest $\mathrm{RBC}$ value was recorded in patients with Progressive Relapsing MS $(3.93 \mathrm{M} / \mu \mathrm{l})$, while the highest in patients with Relapse-Remitting MS $(5.07 \mathrm{M} / \mu \mathrm{l})$.

Other blood parameters did not differ significantly with regard to MS type.

A significant difference $(p=0.0111)$ was also observed in HGB levels for patients with different EDSS scores, as shown in Table 3 and Figure 3.

The next stage of statistical analysis consisted in using Spearman's Rank Correlation test for comparison of blood parameters, duration of illness and EDSS scores. As presented in Table 4, a significant positive correlation was determined for disease duration and platelet count $(\mathrm{R}=0.32$; $\mathrm{p}$ $=0.013)$ as well as for hemoglobin level and EDSS score $(R=0.34 ; p=0.0111)$. A significant positive correlation was determined for duration of disease and patient's disability $(\mathrm{R}=0.41 ; \mathrm{p}=0.048)$.

Table 2. Average values of blood parameters in MS patients with different disease types

\begin{tabular}{|c|c|c|c|c|c|c|c|c|c|}
\hline & 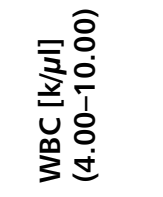 & 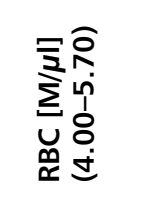 & 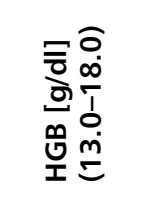 & 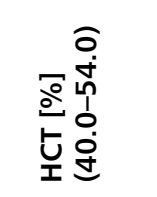 & 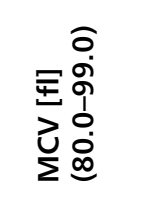 & 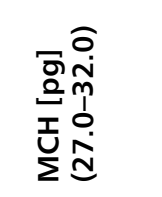 & 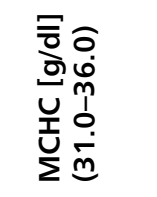 & 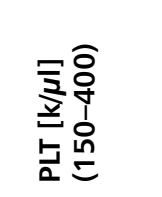 & 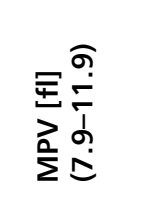 \\
\hline RRMS & 5.91 & 4.74 & 14.0 & 40.7 & 87.9 & 29.9 & 33.3 & 240 & 8.9 \\
\hline $\mathrm{CIS}$ & 6.60 & 5.07 & 14.8 & 44.5 & 86.4 & 29.2 & 33.6 & 237 & 7.8 \\
\hline PPMS & 7.10 & 4.85 & 14.45 & 44.4 & 87.3 & 29.4 & 33.4 & 230.5 & 8.25 \\
\hline RPMS & 6.44 & 3.93 & 11.9 & 35.9 & 91.3 & 30.3 & 33.1 & 215 & 8.0 \\
\hline \multirow[t]{2}{*}{ SPMS } & 6.79 & 5.07 & 14.8 & 44.8 & 88.7 & 29.7 & 33.1 & 206 & 8.9 \\
\hline & $P=0.019$ & $P=0.031$ & $P=0.0759$ & $P=0.087$ & $P=0.661$ & $P=0.651$ & $P=0.950$ & $P=0.305$ & $P=0.183$ \\
\hline
\end{tabular}

Table 3. Hemoglobin level of MS patients with regard to severity of the disease. $X$ - mean, SD — standard deviation, Min - minimum, Max — maximum, Me - Median

\begin{tabular}{|c|c|c|c|c|c|c|c|c|}
\hline \multirow[t]{2}{*}{ EDSS } & \multirow[t]{2}{*}{$\mathbf{N}$} & \multicolumn{5}{|c|}{ HGB } & \multicolumn{2}{|c|}{$\begin{array}{c}\text { Kruskal-Wallis } \\
\text { test }\end{array}$} \\
\hline & & $\mathrm{X}$ & SD & Min. & Max. & Me & H & $\mathbf{P}$ \\
\hline Mild degree of disability (EDSS $1-3,5$ ) & 77 & 13.9 & 1.3 & 9.7 & 16.9 & & & \\
\hline Moderate degree of disability (EDSS 4-5) & 24 & 14.7 & 1.01 & 13.1 & 16.4 & 16.4 & 8.9996 & 0.0111 \\
\hline Severe degree of disability (EDSS 6-9) & 11 & 13.8 & 1.2 & 11.2 & 15.6 & 15.6 & & \\
\hline
\end{tabular}


Significant data is presented in Table 4.

The last stage of statistical analysis of blood parameters included the calculation of absolute values for neutrophil-to-lymphocyte ratio (NLR), monocyte-to-lymphocyte ratio (MLR) and plateletto-lymphocyte ratio (PLR) The analysis was performed using Mann-Whitney U. test and revealed

Table 4. Correlation between blood count values and duration of disease and EDSS

\begin{tabular}{lcc}
\hline Correlation & R value & P value \\
\hline RBC vs. Hgb & 0.84 & 0.030 \\
Duration of disease vs. EDSS & 0.41 & 0.048 \\
PLT vs. duration of disease & 0.32 & 0.013 \\
Hgb vs. EDSS & 0.34 & 0.0111 \\
\hline
\end{tabular}

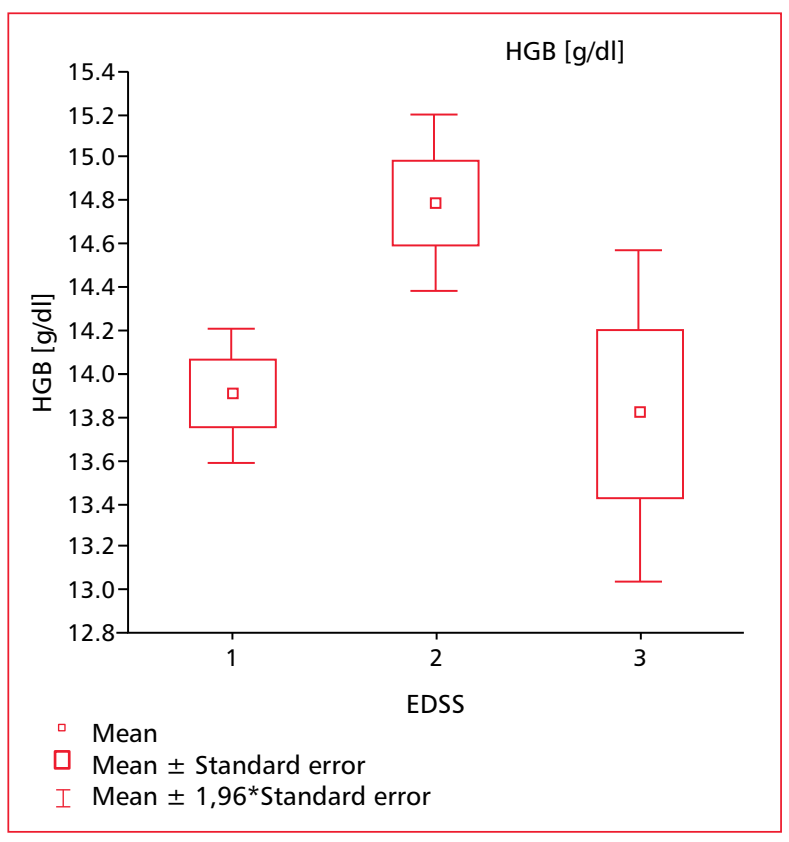

Figure 3. Hemoglobin level of MS patients with various degrees of disability. 1 - light disability (1-3.5 EDSS), 2 - moderate disability (4-5 EDSS), 3 - severe disability (6-9 EDSS). Kruskal-Wallis test no significant differences between NLR, MLR and PLR values in the study group as compared to control group. The NLR value was significantly higher $(\mathrm{p}=0.0201)$ in remission than in relapse, as shown in Figure 4.

Comparative analysis of NLR, MLR and PLR values of patients with various types of MS was performed with ANOVA Kruskal-Wallis test. A significant difference $(\mathrm{p}=0.0065)$ was found for NLR values in patients with various types of MS. The post-hoc Tukey's test for unequal group sizes demonstrated significant differences of NLR value between patients with RRSM and PPSM ( $=0.0003)$ and between patients with PPSM and control group $(\mathrm{p}=0.0019)$ (Table 5).

No statistically significant differences were found for NLR, MLR, PLR values with regard to EDSS score And there was no correlation between these values and age and disease duration.

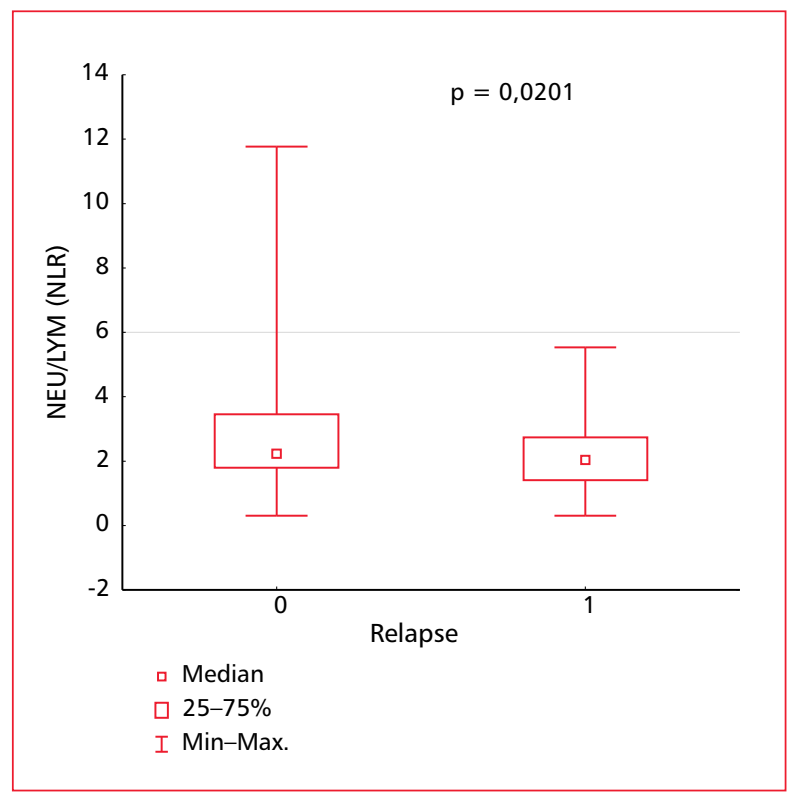

Figure 4. NLR in MS patients in remission (0) and in relapse (1)

Table 5. P-significance coefficient for the NLR indicator in individual groups of patients with various forms of MS. Tukey's RIR test

\begin{tabular}{|c|c|c|c|c|c|}
\hline \multicolumn{6}{|c|}{ Tukey's test for unequal group sizes } \\
\hline MS type & (1) $M=2.0545$ & (2) $M=2.5868$ & (3) $M=3.6004$ & (4) $M=2.4093$ & (5) $\mathrm{M}=2.2323$ \\
\hline RRMS (1) & & 0.7087 & 0.0003 & 0.9827 & 0.9191 \\
\hline $\operatorname{CIS}(2)$ & 0.7087 & & 0.1094 & 0.9989 & 0.9159 \\
\hline PPMS (3) & 0.0003 & 0.1094 & & 0.3583 & 0.0019 \\
\hline SPMS (4) & 0.9827 & 0.9988 & 0.3583 & & 0.9989 \\
\hline Control group 5) & 0.9191 & 0.9159 & 0.0019 & 0.9989 & \\
\hline
\end{tabular}




\section{Conclusions}

1. Analysis of blood parameters reveals significant differences in red blood cells of MS patients as compared to controls as well as among patients with various MS types. WBC is higher in MS patients than in control group due to increased activity of B-lymphocytes and neutrophils in pathogenesis of the disease. B-lymphocytes regulate the autoimmune response and participate in pathological processes in the CNS [9, 25].

2. The statistically significant differences $(p=0.001)$ in hemoglobin levels and RBC values among patients with different EDSS scores may be ascribed to MS as a chronic disease and anemia in some patients.

3. The statistically significant differences in platelet count between the study group and controls indicate that platelets are highly active in some MS patients. Furthermore, by virtue of being adhesive to the vascular endothelium, they are a rich source of anti-inflammatory factors involved in the immunological response of blood vessels [26]. The significantly lower NLR value in relapse as compared to that in remission suggest the participation of inflammatory cells (mostly lymphocytes) in the demyelination process. Lower NLR value in RRMS patients are further confirmation.

\section{Discussion}

The regenerative capacity of the nervous system is limited therefore any MS related pathological lesions in the brain lead to malfunction of the CNS. In the course of the disease there occurs a gradual disappearance of myelin sheath and oligodendrocytes. Typically the disease is characterized initially by episodes of reversible neurological deficits, which is often followed by progressive neurological deterioration over time (relapse/ /remission). With each relapse, the neurological symptoms get worse. Basic blood parameters of MS patients differ from those of healthy volunteers. Furthermore, there occur differences in blood parameters among patients with different MS types. In their study Akaishi et al. [27] assessed peripheral whole blood parameters of MS patients to conclude that the number of peripheral monocytes in the early MS stage correlates with the clinical stage of the disease. This is not the case with other blood parameters. Bar-Or et al. [28] have demonstrated higher levels of two metalloproteinases (MMPs) expressed by monocytes of MS patients as compared to healthy population. The results indicate that monocytes are significant markers of the CNS inflammation. In the future, this parameter may have significant prognostic value for MS diagnosis.

In their search for a simple, low cost and repetitive biomarker Demirci et al. have demonstrated that NLR values in MS patients are significantly higher than in healthy people. Moreover, the NLR values were higher in relapse than in remission. They claim that NLR might be an important MS prognostic factor [23]. However, the authors of this study have arrived at completely different results which may be the result of different selection criteria for study cohort and control group, unequal group sizes and low sensitivity of the NLR indicator.

Moreover, the degree of disability is closely associated with disease duration. MS is a chronic disease, therefore patients who have suffered for $15-20$ years have substantially reduced mobility and require continuous treatment [29].

In their study of 44 natalizumab-treated MS patients Briedl et al. [30] demonstrated a significant increase in lymphocyte, monocyte and eosinophile counts. Pastuszak et al. [31] confirmed the effect of anthracycline antibiotic mitoxantrone (MTX) on blood count. In their study of 70 MTX-treated MS patients, they demonstrated a decrease of leukocyte, erythrocyte and thrombocyte counts. In the case of erythrocyte count the decrease was statistically significant.

To sum up, there are statistically significant differences in blood parameters between MS patients and healthy volunteers. This may be affected by many factors such as disease duration, type of MS or medication. Currently, there is an ongoing search for new markers and novel diagnostic tests that would accelerate MS diagnosis and facilitate treatment.

\section{References}

1. Sadovnick AD. Genetic epidemiology of multiple sclerosis: a survey. Ann Neurol. 1994; 36 Suppl 2: S194-S203, indexed in Pubmed: 7998788.

2. Ebers GC. Prognostic factors for multiple sclerosis: the importance of natural history studies. J Neurol. 2005; 252 Suppl 3: iii15-iii20, doi: 10.1007/s00415-005-2012-4, indexed in Pubmed: 16170495.

3. Amato MP, Ponziani G, Bartolozzi ML, et al. A prospective study on the natural history of multiple sclerosis: clues to the conduct and interpretation of clinical trials. J Neurol Sci. 1999; 168(2): 96-106, doi: 10.1016/s0022-510x(99)00143-4, indexed in Pubmed: 10526190.

4. Potemkowski A. Stwardnienie rozsiane w świecie i w Polsce ocena epidemiologiczna. Aktualn Neurol. 2009; 9: 91-97. 
5. Bradburn J, Broughton S, Chadwick D. Royal College of Physicians of London: Multiple sclerosis. National clinical guideline for diagnosis and management in primary and secondary care. 2004; 8: 39-46.

6. Kantarci $\mathrm{OH}$, Weinshenker BG. Natural history of multiple sclerosis. Neurol Clin. 2005; 23(1): 17-38, v, doi: 10.1016/j. ncl.2004.10.002, indexed in Pubmed: 15661086.

7. Rosiak K, Zagożdżon P. Czynniki środowiskowe w epidemiologii stwardnienia rozsianego. Probl Hig Epidemiol. 2012; 93(4): 627-631.

8. Sadovnick AD, Ebers GC. Epidemiology of multiple sclerosis: a critical overview. Can J Neurol Sci. 1993; 20(1): 17-29, doi: 10.1017/s0317167100047351, indexed in Pubmed: 8467424.

9. Shabab T, Khanabdali R, Moghadamtousi SZ, et al. Neuroinflammation pathways: a general review. Int J Neurosci. 2017; 127(7): 624-633, doi: 10.1080/00207454.2016.1212854, indexed in Pubmed: 27412492.

10. Ebers M. Natural history of multiple sclerosis. W: Compston A. (red. ) Mc Alpine's Multiple Sclerosis, New York, Churchill Livingstone; 1998: 191-221.

11. Ransohoff RM. How neuroinflammation contributes to neurodegeneration. Science. 2016; 353(6301): 777-783, doi: 10.1126/ science.aag2590, indexed in Pubmed: 27540165.

12. Freal JE, Kraft GH, Coryell JK. Symptomatic fatigue in multiple sclerosis. Arch Phys Med Rehabil. 1984; 65(3): 135-138, indexed in Pubmed: 6703889.

13. Miller DH, Hornabrook RW, Purdie G. The natural history of multiple sclerosis: a regional study with some longitudinal data. J Neurol Neurosurg Psychiatry. 1992; 55(5): 341-346, doi: 10.1136/jnnp.55.5.341, indexed in Pubmed: 1602305.

14. Smelkowska A, Wilkiewicz M, Grabowska-Fudala B, et al. Course of Care of a Multiple Sclerosis Patient in the Context of Pharmacotherapy. PNN 2015; 4: 130-137.

15. Stuart WH. Clinical management of multiple sclerosis: the treatment paradigm and issues of patient management. J Manag Care Pharm. 2004; 10(3 Suppl B): S19-S25, indexed in Pubmed: 15253686.

16. Lublin FD, Reingold SC, Cohen JA, et al. Defining the clinical course of multiple sclerosis: the 2013 revisions. Neurology. 2014; 83(3): 278-286, doi: 10.1212/WNL.0000000000000560, indexed in Pubmed: 24871874.

17. Selter RC, Hemmer B. Update on immunopathogenesis and immunotherapy in multiple sclerosis. Immunotargets Ther. 2013; 2 : 21-30, doi: 10.2147/ITT.S31813, indexed in Pubmed: 27471685.

18. Wender M. Biomarkery w stwardnieniu rozsianym. Aktualn Neurol. 2009; 9 (2), p. : 109-115.

19. Thompson AJ, Banwell BL, Barkhof F, et al. Diagnosis of multiple sclerosis: 2017 revisions of the McDonald criteria. Lancet Neu- rol. 2018; 17(2): 162-173, doi: 10.1016/S1474-4422(17)30470-2, indexed in Pubmed: 29275977.

20. Polman C, Reingold S, Edan G, et al. Diagnostic criteria for multiple sclerosis: 2005 revisions to the "McDonald Criteria". Annals of Neurology. 2005; 58(6): 840-846, doi: 10.1002/ana.20703.

21. Housley WJ, Pitt D, Hafler DA. Biomarkers in multiple sclerosis. Clin Immunol. 2015; 161(1): 51-58, doi: 10.1016/j. clim.2015.06.015, indexed in Pubmed: 26143623.

22. Huang Y, Ying Z, Quan W, et al. The clinical significance of neutrophil-to-lymphocyte ratio and monocyte-to-lymphocyte ratio in Guillain-Barré syndrome. Int J Neurosci. 2018; 128(8): 729-735, doi: 10.1080/00207454.2017.1418342, indexed in Pubmed: 29251087.

23. Demirci S, Demirci S, Kutluhan S, et al. The clinical significance of the neutrophil-to-lymphocyte ratio in multiple sclerosis. Int J Neurosci. 2016; 126(8): 700-706, doi: 10.3109/00207454.2015.1050492, indexed in Pubmed: 26000934.

24. Qin B, Ma N, Tang Q, et al. Neutrophil to lymphocyte ratio (NLR) and platelet to lymphocyte ratio (PLR) were useful markers in assessment of inflammatory response and disease activity in SLE patients. Mod Rheumatol. 2016; 26(3): 372-376, doi: 10.3109/14397595.2015.1091136, indexed in Pubmed: 26403379.

25. Wekerle H. B cells in multiple sclerosis. Autoimmunity. 2017; 50(1): 57-60, doi: 10.1080/08916934.2017.1281914, indexed in Pubmed: 28166681.

26. Dziedzic A, Bijak M. Interactions between platelets and leukocytes in pathogenesis of multiple sclerosis. Adv Clin Exp Med. 2019; 28(2): 277-285, doi: 10.17219/acem/83588, indexed in Pubmed: 30411550.

27. Akaishi T, Takahashi T, Nakashima I. Peripheral blood monocyte count at onset may affect the prognosis in multiple sclerosis. J Neuroimmunol. 2018; 319: 37-40, doi: 10.1016/j.jneuroim.2018.03.016, indexed in Pubmed: 29685288.

28. Bar-Or A, Nuttall RK, Duddy M, et al. Analyses of all matrix metalloproteinase members in leukocytes emphasize monocytes as major inflammatory mediators in multiple sclerosis. Brain. 2003; 126(Pt 12): 2738-2749, doi: 10.1093/brain/awg285, indexed in Pubmed: 14506071.

29. Bonek R, Maciejek Z. Naturalny przebieg stwardnienia rozsianego. Aktualn Neurol. 2009; 9(2): 116-125.

30. Bridel C, Beauverd Y, Samii K, et al. Hematologic modifications in natalizumab-treated multiple sclerosis patients: An 18-month longitudinal study. Neurol Neuroimmunol Neuroinflamm. 2015; 2(4): e123, doi: 10.1212/NXI.0000000000000123, indexed in Pubmed: 26140281.

31. Pastuszak Z, Stepien A, Tomczykiewicz K, et al. Blood count in patients with multiple sclerosis treated with mitoxantrone in short time observation. Acta Pol Pharm. 2016; 73(5): 1369-1373, indexed in Pubmed: 29638077. 